\title{
A statistical study of wave propagation in coronal holes
}

\author{
E. O'Shea ${ }^{1}$, D. Banerjee ${ }^{2}$, and J. G. Doyle ${ }^{1}$ \\ 1 Armagh Observatory, College Hill, Armagh BT61 9DG, N. Ireland \\ e-mail: [eos; jgd] @arm.ac.uk \\ 2 Indian Institute of Astrophysics, II Block, Koramangala, Bangalore 560 034, India \\ e-mail: dipu@iiap.res.in \\ Received 11 May 2006 / Accepted 23 September 2006
}

\begin{abstract}
Aims. To find evidence for propagating magnetoacoustic waves in equatorial and polar coronal hole locations.

Methods. Using temporal series data from the Coronal Diagnostic Spectrometer (CDS) on SOHO, we study oscillations found in radiant flux and velocity measurements from transition region (O v 629) and coronal lines (Mg X 624, Si XII 520). We use Fourier techniques to measure phase delays between flux ("intensity") oscillations and between velocity oscillations of different transition region-corona and corona-corona line pairs. We also measure the phase delays between flux and velocity oscillations (I-V) in the three spectral lines investigated.

Results. We find outwardly propagating slow magnetoacoustic waves in both of the coronal hole regions studied. The propagation speeds are found to be lower than those found in off-limb locations. We find evidence for a resonant cavity or "Doppler" effect, whereby the measured phases are present at fixed integer intervals of $f / 4\left(90^{\circ}\right.$ of phase) and $3 f / 8\left(135^{\circ}\right.$ of phase) instead of the expected interval of $f$ or $360^{\circ}$. We find, in addition, from the $\mathrm{I}-\mathrm{V}$ phases, evidence for standing waves at coronal temperatures in the lines of Mg X 624 and Si XII 520. Correlations are found between the locations where the phases are measured and localised brightenings in both equatorial and polar coronal holes. This suggests that the slow magnetoacoustic waves are originating preferentially from bright areas within the coronal holes which we take to be the locations of concentrated magnetic field (loops, bright points). Finally, we find evidence that in these bright regions along the slit, the measured phases tend to occur at a spectrum of frequencies, perhaps suggesting the presence of discrete propagating wave packets.

Conclusions. We conclude that propagating slow magnetoacoustic waves are present in equatorial and polar coronal hole locations and that they occur preferentially in bright regions that are associated with magnetic field concentrations in the form of loops or bright points. In addition, we conclude that some resonant cavity effect is affecting the propagating waves, perhaps resulting in the standing waves that are found at coronal temperatures.
\end{abstract}

Key words. Sun: UV radiation - Sun: transition region - Sun: corona - Sun: oscillations - Sun: atmosphere

\section{Introduction}

Coronal holes are regions of cool and low density plasma that are "dark" at coronal temperatures (Munro \& Withbroe 1972). During the years of the solar minimum these coronal holes are confined to the Sun's polar regions, while at solar maximum they can be found at all latitudes, usually associated with remnant active regions, as so-called equatorial coronal holes. The predominantly unipolar magnetic fields in coronal hole regions are thought likely to give rise to the fast solar wind (Krieger et al. 1972). Recently, it has been discovered that the fast solar wind originates from coronal funnels in the polar regions ( $\mathrm{Tu}$ et al. 2005). Different studies have found evidence for outflows (blueshifts) of typically $10 \mathrm{~km} \mathrm{~s}^{-1}$ in both polar (Wilhelm et al. 2000) and equatorial (Xia et al. 2004) coronal holes at transition region temperatures. To reach the high speeds of some hundreds of $\mathrm{km} \mathrm{s}^{-1}$ (Teriaca et al. 2003), at radial distances of more than $1.5 R_{\odot}$, found in the fast solar wind, it is clear that some additional mechanism, apart from thermal conduction, is necessary (see Davila 1985). The funnel model proposed by Tu et al. 2005 invokes the idea of reconnection and the release of Alfvén waves. We note that Ofman \& Davila (1997) discuss a nonlinear model whereby compressional MHD waves are generated via Alfvén waves and that it is these MHD waves that are one of the sources of the additional acceleration. A number of studies (Ofman et al. 1997; Ofman et al. 2000; Banerjee et al. 2001) have measured oscillations in coronal holes in the polar regions of the Sun. All of these studies point to the presence of compressional waves, thought to be slow magnetoacoustic waves as found by DeForest \& Gurman (1998) and O'Shea et al. (2006). In this work we look for evidence of these compressional waves in polar and equatorial coronal hole regions and seek to positively identify them by means of their propagation speeds.

\section{Observations and data reduction}

For these observations we have used the normal incidence spectrometer (NIS), which is one of the components of the Coronal Diagnostic Spectrometer (CDS) on board the Solar and Heliospheric Observatory (SOHO), see Harrison et al. (1995).

The temporal series SER150W sequence was run during December 2002 in both a Northern and Southern coronal hole locations as well as two equatorial coronal hole locations (see Table 1). Figures 1 and 2 shows the slit locations for the CDS datasets, over-plotted on EIT $171\left(\log T \approx 1.3 \times 10^{6} \mathrm{~K}\right)$ and EIT 284 images $\left(\log T \approx 2 \times 10^{6} \mathrm{~K}\right)$, which were observed at times approximate to those of the CDS datasets. 

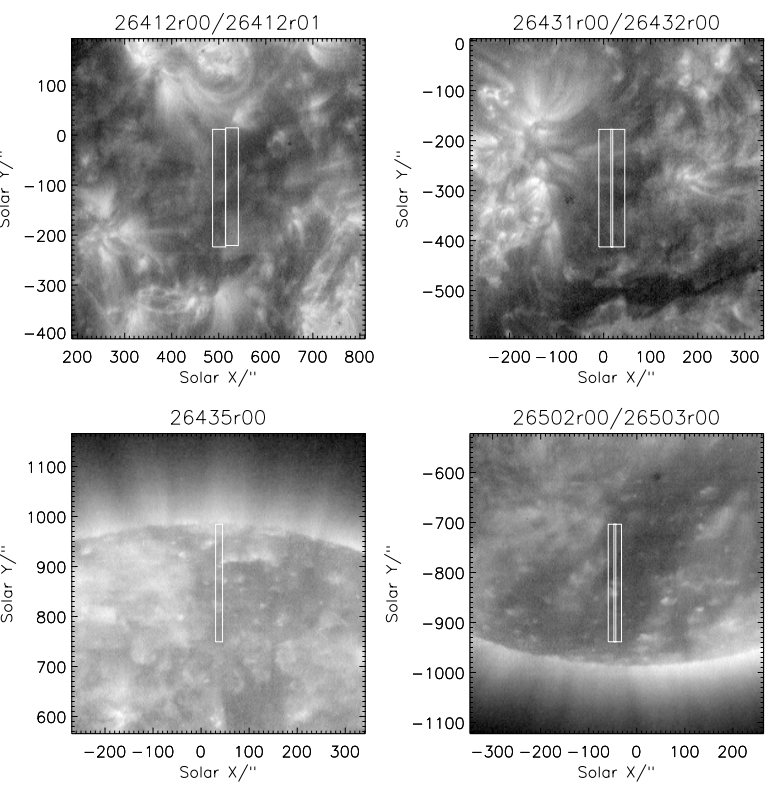

Fig. 1. (Top left) EIT 171 image taken at 13:00 UTC on 7/12/02 showing the total area traversed by the $26412 \mathrm{r} 00$ slit (on the left) and the 26412 r01 slit (on the right) during the period of the observations. (Top right) EIT 171 image taken at 19:00 UTC on 10/12/02 showing the area traversed by the 26431r00 slit (on the right) and the 26432r00 slit (on the left). (Bottom left) EIT 171 image taken at 07:00 UTC on the $11 / 12 / 02$ showing the area traversed by the $26435 \mathrm{r} 00$ slit. (Bottom right) EIT 171 image taken at 19:00 UTC on the 20/12/02 showing the area traversed by the $26502 \mathrm{r} 00$ slit (on the right) and 26503r00 (on the left).

Table 1. SER150W datasets obtained using the $4 " \times 240^{\prime \prime}$ CDS slit and an exposure time of 60s during December 2002.

\begin{tabular}{llcl}
\hline \hline Date & Dataset & Pointing $(X, Y)$ & Start/End (UTC) \\
\hline $07 / 12$ & $26412 \mathrm{r} 00$ & $507,-104$ & $12: 22 / 15: 13$ \\
$07 / 12$ & $26412 \mathrm{r} 01$ & $557,-101$ & $15: 13 / 18: 03$ \\
$10 / 12$ & $26431 \mathrm{r} 00$ & $20,-293$ & $16: 27 / 19: 17$ \\
$10 / 12$ & $26432 \mathrm{r} 00$ & $20,-293$ & $19: 18 / 22: 08$ \\
$11 / 12$ & $26435 \mathrm{r} 00$ & 41,869 & $06: 34 / 09: 24$ \\
$20 / 12$ & $26502 \mathrm{r} 00$ & $-38,-819$ & $18: 00 / 20: 51$ \\
$20 / 12$ & $26503 \mathrm{r} 00$ & $-38,-819$ & $20: 51 / 23: 41$ \\
\hline
\end{tabular}

Data were obtained for 11 transition region and coronal lines. However, here we shall only discuss four of these; the transition region line of $\mathrm{O} v 629.73 \AA\left(\approx 2.5 \times 10^{5} \mathrm{~K}\right)$ and the coronal lines of $\mathrm{Mg}$ X $609.79,624.94 \AA\left(\approx 1.25 \times 10^{6} \mathrm{~K}\right)$ and Si XII $520.67 \AA\left(\approx 2.5 \times 10^{6} \mathrm{~K}\right)$. Note that we shall henceforth refer to the lines without the following decimal places, e.g., 629 in place of 629.73. The data were reduced using the latest versions of the standard CDS routines ${ }^{1}$. Before fitting the lines with a single Gaussian, and in order to increase the signal-to-noise ratio, we binned by 2 along the 143 pixel slit to produce 70 usable pixels $\left(4^{\prime \prime} \times 3.36^{\prime \prime}\right)$ in $Y$.

All line-of-sight (LOS) velocities in this work are measured relative to an "averaged" fitted line, obtained by summing together all the individual lines at each pixel position along the slit (70) and at each time frame (150). The measured wavelength position of this "averaged" line is then taken to be the reference wavelength, and all velocities are measured relative to it. In this work we, therefore, make use of relative velocity values.

\footnotetext{
${ }^{1}$ http://solar.bnsc.rl.ac.uk/software/uguide/ uguide. shtml
}
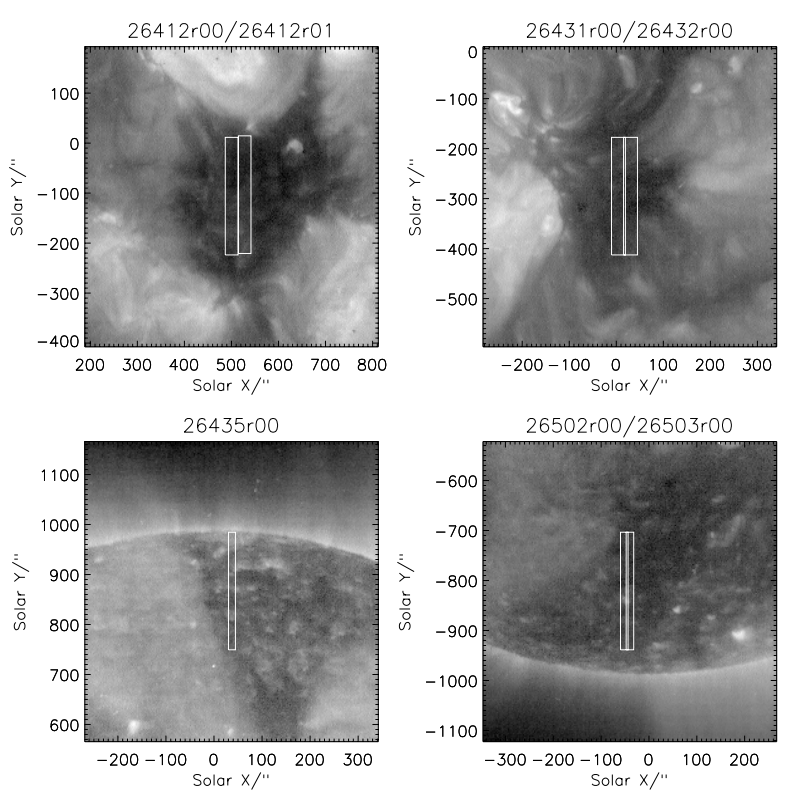

Fig. 2. (Top left) EIT 284 image taken at 13:06 UTC on 7/12/02 showing the total area traversed by the $26412 \mathrm{r} 00$ (on the left) and the 26412r01 slit (on the right) during the period of the observations. (Top right) EIT 171 image taken at 19:06 UTC on 10/12/02 showing the area traversed by the $26431 \mathrm{r} 00$ slit (on the right) and the 26432r00 slit (on the left). (Bottom left) EIT 171 image taken at 07:06 UTC on the 11/12/02 showing the area traversed by the $26435 \mathrm{r} 00$ slit. (Bottom right) EIT $171 \mathrm{im}-$ age taken at 19:06 UTC on the 20/12/02 showing the area traversed by the 26502r00 slit (on the right) and 26503r00 (on the left).

As reported in Table 1, the exposure time for each of the datasets was $60 \mathrm{~s}$, leading to a cadence of $\approx 68 \mathrm{~s}$ in each case. For each of the 8 datasets listed in Table 1, 150 time frames were obtained in a sit-and-stare study, that is, the CDS slit was left at the same pointing over the whole observation time. The total observation time, therefore, for each dataset is $150 \times 68 \mathrm{~s} \approx 1.02 \times 10^{4} \mathrm{~s} \approx 170 \mathrm{~min}$, the frequency resolution is $\approx 9.80 \times 10^{-2} \mathrm{mHz}$ and the Nyquist frequency is $\approx 7.35 \mathrm{mHz}$. In the following (Fourier) analysis, phase delays for each dataset will be measured at all frequencies up to the Nyquist frequency, at steps dictated by the frequency resolution. We note that as we are observing on-disk rotation effects can become important. We estimate that in the polar coronal hole observations the rotation causes a spreading of $\approx 0.3 \mathrm{mHz}$ in the measured frequencies of oscillation. In the equatorial coronal hole observations we estimate the effect to be of the order of $\approx 0.6 \mathrm{mHz}$. Therefore, we do not take account of any oscillation frequencies measured below these values in either location.

\section{Results}

Discussing the equatorial coronal hole datasets first, and following the techniques outlined in O'Shea et al. $2006^{2}$, we show in Fig. 3 the combined phase delay results from the $26412 \mathrm{r} 00$ and 26412 r01 datasets. The phases are calculated here for the three line pairs, O V 629-Mg X 624, O v 629-Si XII 520 and Mg X 624-Si XII 520. Note that we consider it acceptable to combine the results of these two datasets in order to improve the statistics as both datasets are approximately co-spatial and belong to the same coronal hole region. We are looking for the global presence of oscillations so this summing should, in any case, not be

\footnotetext{
${ }^{2}$ http://www.arm.ac.uk/preprints
} 


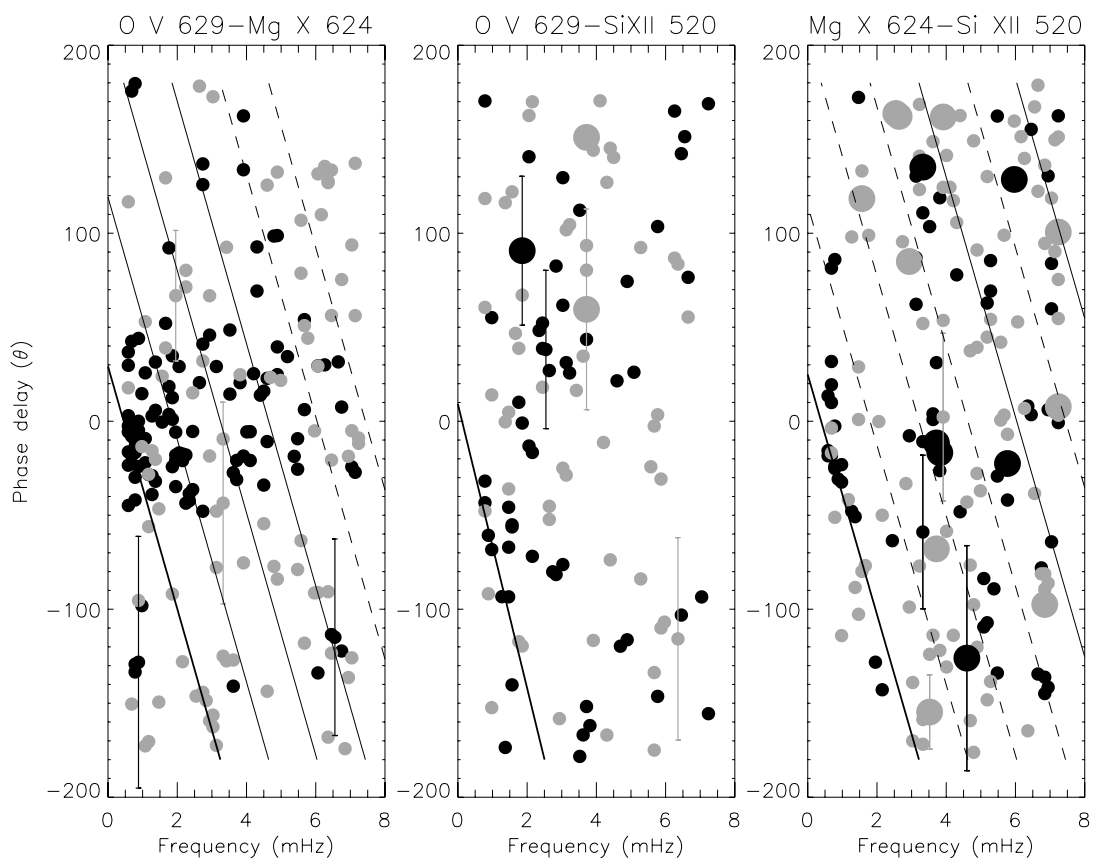

Fig. 3. Phase delays measured between the oscillations in the different line pairs, as labelled, from the combined 26412r00 and 26412r01 datasets. Radiant flux oscillations are shown as the black circle symbols while L.O.S. velocities are shown as the grey circle symbols. Phase delays measured at the $99 \%$ significance level are indicated by the larger symbols, while the smaller symbols represent measurements at the $95 \%$ significance level. Typical uncertainties in the estimated phase delays are indicated by the error bars. Over-plotted on each plot are thick black lines passing through the zero point of phase, corresponding to fixed time delays. On the plots of the O v 629-Mg X 624 and Mg X 624-Si XII 520 line pairs are other lines (solid and dashed) spaced at $f / 4$ frequency intervals or $90^{\circ}$ of phase. Dashed lines refer to intervals where there is statistical uncertainty as to the presence of a line at the expected $90^{\circ}$ spacing. relevant to our results. In fact, looking at the locations of these datasets in Figs. 1 and 2, it can be seen that they are both located in a small coronal hole surrounded by a number of active regions. As in O'Shea et al. (2006), we can see that the phases in Fig. 3 are distributed between $-180^{\circ}$ and $180^{\circ}$. Again, as in O'Shea et al. (2006) we might expect the phases to line up along sloping parallel lines if there are fixed time delays present between the oscillations in the different lines.

To investigate whether there is any statistical evidence for such a linear distribution of phases, we first fit the phase delay measurements lying along a line passing through the zero point of phase. This fitted line is shown in Fig. 3 as the thicker black sloping line. The technique used to produce this fitted line is explained in some detail in O'Shea et al. (2006), so we will only briefly describe it here. Firstly, an appropriate time delay for each line pair is obtained (through trial and error or otherwise) and used with Eq. (1) (see later) to draw a straight line through the data. Those datapoints lying within a set distance $(\sigma / 2$ or $1 \sigma)$ of the line at each frequency location are chosen and fitted with a 1 st order polynomial to accurately measure the slope $(2 \pi T)$ and, hence, the time delay. Using this time delay and Eq. (1), a new straight line is drawn through the data and the process of choosing datapoints and measuring slopes repeated until such time as there is convergence of the time delay to a consistent value. In a difference to the technique used in the O'Shea et al. (2006) paper, here we fit all those points that lie within $1 \sigma$ of the initial trial-and-error "fit" and not $\sigma / 2$, due to the reduced number of phase points available to us in these plots. Often, an obvious linear distribution in the data is not immediately visible by eye, due to a reduced number of points, and the initial trialand-error "fit" is, of necessity, a crude estimation, e.g., in the case of the O V 629-Si XII 520 line pair here. In the case of this line pair, the lack of phase measurements contributes to a large uncertainty in the fitted line and in the subsequent measurement of the slope $(2 \pi T)$ and, hence, time delay. In short, in this work we will make the assumption that a linear distribution is present, as predicted by the phase equation (Eq. (1)), and show, through the use of histograms that parallel linear rows of phase measurements, indicating fixed time delays, are present.
From the fitted lines to the phase measurements in Fig. 3, we estimate the slopes and, therefore, the time delays between the different line pairs to be $179 \pm 26 \mathrm{~s}(f \approx 5.58 \mathrm{mHz})$ for $\mathrm{OV}$ $629-\mathrm{Mg}$ X $624,210 \pm 108 \mathrm{~s}(f \approx 4.76 \mathrm{mHz})$ for O V 629-Si XII 520 and $177 \pm 39 \mathrm{~s}(f \approx 5.65 \mathrm{mHz})$ for $\mathrm{Mg}$ X 624-Si XII 520 . We note that O V 629-Si XII 520 shows very large uncertainties in the measured time delays, as expected, due to the reduced number of points making up the fit.

Using the fitted lines in Fig. 3, and shifting the phase measurements to the horizontal, histograms with appropriate confidence levels can be constructed, again as in O'Shea et al. (2006). The results of this are shown in Fig. 4. We note that the bin size used in these histograms depends on the error in the y-intercept point of the fitted lines for each of the line pairs. This ensures that any inaccuracies in the fit of the lines in Fig. 3, e.g., large uncertainties due to a limited number of points, will be accounted for in the histograms. For example, variations in the slope of the fitted line, due to these uncertainties, could potentially produce different histogram distributions when the fitted line is used to shift the measured phases up to the horizontal. Using the appropriate bin size means that these uncertainties are accounted for within the broader histogram bins. The bin size is taken to be $10^{\circ}$ for the O v 629-Mg X 624 line pair, 50 for the O V 629-Si XII 520 line pair and $20^{\circ}$ for the $\mathrm{Mg} \mathrm{X} 624-\mathrm{Si}$ XII 520 line pair.

From Fig. 4, one can see that the phases for the Ov 629Mg X 624 line pair (left panel) are distributed at phase spacings of $90^{\circ}$. For example, there are peaks above the $90 \%$ confidence level $0^{\circ}$ and $90^{\circ}$, while at $\approx 180^{\circ}$ and $\approx 270^{\circ}$, the highest peaks are present above the $90 \%$ confidence level. We consider, in this case, that the peaks at $170^{\circ}$ and $200^{\circ}$ form part of a spread of phase measurements around the expected $180^{\circ}$ point. This spread of points is understandable considering the large errors, $>50-60^{\circ}$, associated with each of the phase measurements in Fig. 3. Similarly, we also consider the peak at $280^{\circ}$ to be due to the spreading of phase measurements around the $270^{\circ}$ point. At higher phase angles of $>270^{\circ}$ the fixed $90^{\circ}$ separation breaks down, due to a more limited number of points, and we cannot anymore say whether there is still a fixed separation present. We note, however, that there are still significant peaks above the $95 \%$ 

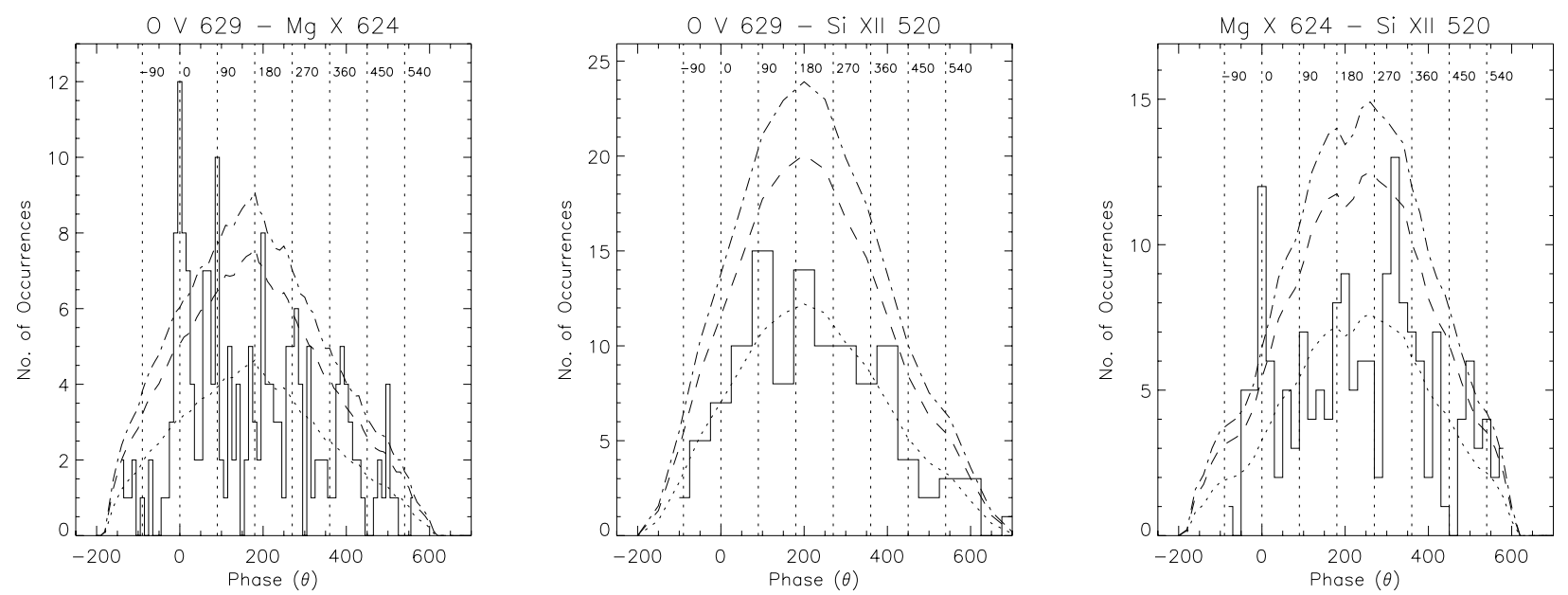

Fig. 4. Histograms, for the three line pairs as labelled, showing the distribution of phase as a function of frequency in the combined $26412 \mathrm{r} 00 / 26412 \mathrm{r} 01$ datasets. Dotted vertical lines indicate phase intervals of $90^{\circ}$. Overplotted on the histograms as dotted, dashed and dot-dashed horizontal lines are the result of Monte Carlo simulations with 5000 permutations. The dotted lines show the expected noise noise distribution, i.e., the $1 \sigma, 68.3 \%$ confidence level; the dashed lines the $90 \%$ confidence level, i.e., the $\approx 1.6 \sigma$ level; and the dot-dashed lines the $95 \%$ confidence level, i.e., the $\approx 2 \sigma$ level.

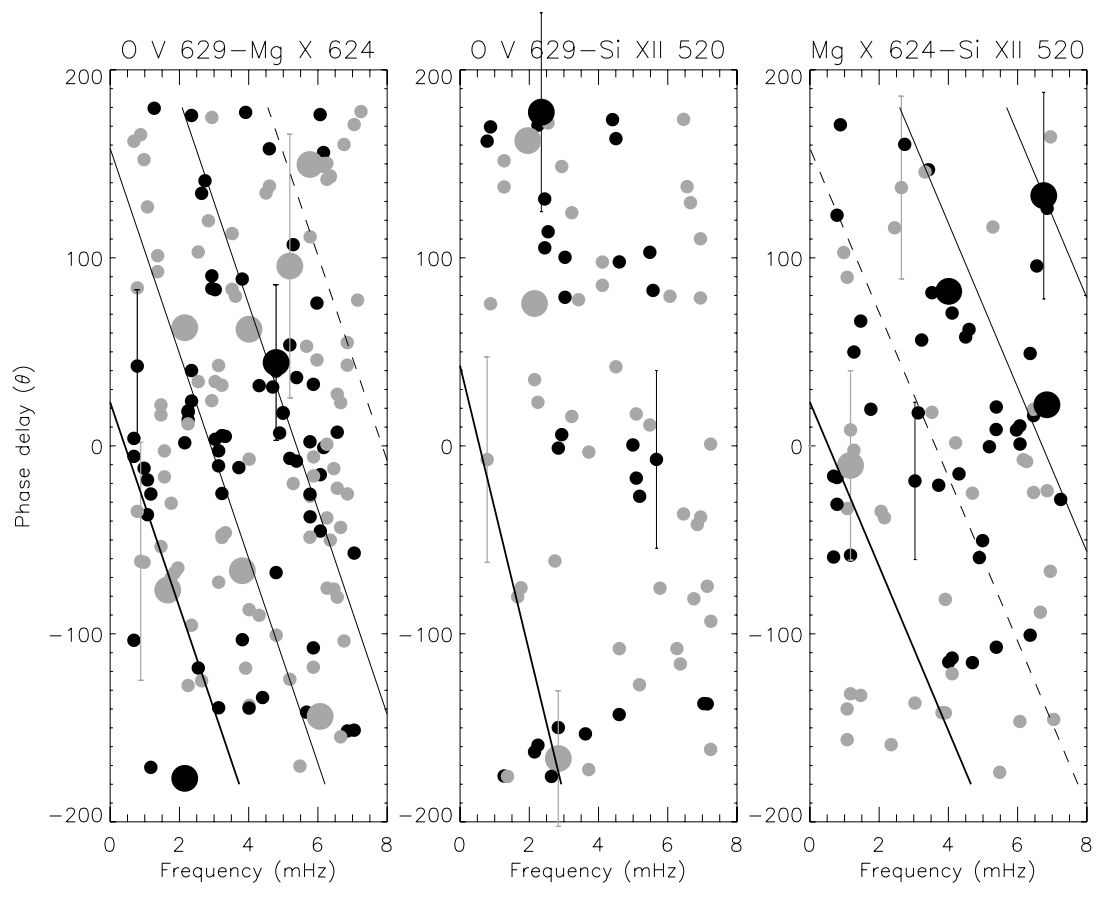

Fig. 5. The same as Fig. 3 presented here for the combined datasets $26431 \mathrm{r} 00$ and $26432 \mathrm{r} 00$. Here, on the plots of the OV 629-Mg X 624 and Mg X 624-Si XII 520 line pairs, the solid and dashed lines are spaced at $3 f / 8$ frequency intervals or $135^{\circ}$ of phase. As before, the dashed lines refer to intervals where there is statistical uncertainty as to the presence of a line at the expected $135^{\circ}$ spacing. significance level at $390^{\circ}$ and $500^{\circ}$, but that these are at distances of $30^{\circ}$ and $50^{\circ}$ from the expected positions of $360^{\circ}$ and $450^{\circ}$, respectively, assuming a fixed $90^{\circ}$ separation. In Fig. 3, we plot thin black lines at $90^{\circ}$ spacings, at angles corresponding to the fixed time delay of $179 \pm 26 \mathrm{~s}$. At the locations where the strict fixed $90^{\circ}$ separation breaks down, as discussed above, we plot the sloping lines as dashes to indicate this, e.g., the first four peaks in Fig. 4 were significant and so the first four sloping lines in Fig. 3 are plotted as continuous black lines, while the subsequent lines are plotted with dashes to indicate that subsequent peaks in Fig. 4 were not significant.

Carrying out the same procedure on the other two line pairs, we get, for O V 629-Si XII 520, the histogram in Fig. 4 (middle panel) and, for Mg X 624-Si XII 520, the histogram in Fig. 4 (right panel). In the O V 629-Si XII 520 histogram no peak is present above either the $90 \%$ or $95 \%$ confidence levels. For this reason, no additional sloping lines are plotted on the phase measurement of this line pair in Fig. 3, apart from the thick black line fit. In the histogram of the Mg X 624-Si XII 520 line pair, there are peaks spaced at $90^{\circ}$ above the $90 \%$ confidence level at $0^{\circ}, 320^{\circ}$ and $540^{\circ}$. We assume, as for the $\mathrm{O}$ v 629-Mg X 624 line pair, that the peak at $320^{\circ}$ is due to the scattered phase values expected at the $360^{\circ}$ location. We note, however, that the peaks at $90^{\circ}, 180^{\circ}, 270^{\circ}$ and $450^{\circ}$ are either at the level of noise or only marginally above it. In this case, as for the O v 629-Si XII 520 line pair, it is not possible, therefore, to come to any conclusion about the presence of a fixed $90^{\circ}$ phase spacing. We plot, however, in Fig. 3, for the Mg X 624-Si XII 520 line pair, solid sloping lines at spacings of $90^{\circ}$ at phase locations where the peaks in the histogram in Fig. 4 are above the $90 \%$ confidence level and dashed sloping lines elsewhere.

Following the procedure undertaken in Figs. 3 and 4, we plot in Figs. 5 and 6 the combined results of the other equatorial coronal hole datasets, 26431r00 and 26432r00. As can be seen from 

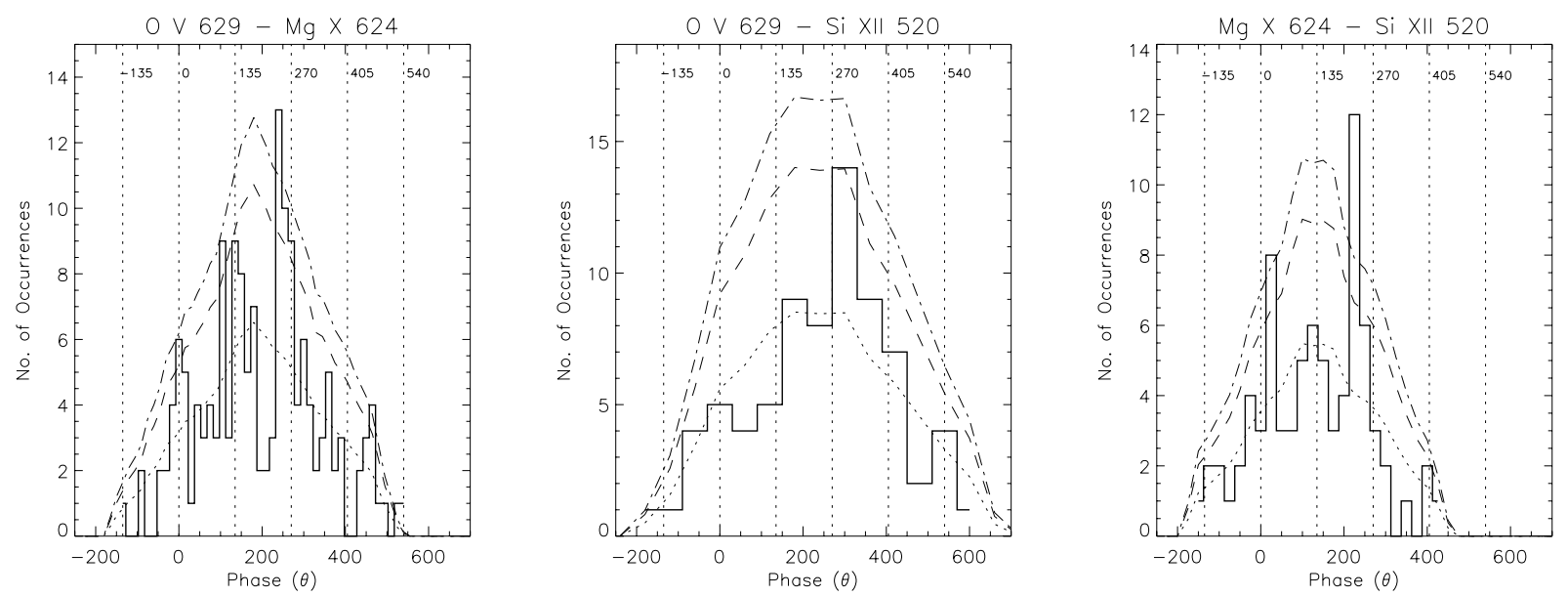

Fig. 6. The same as Fig. 4 presented here for the combined datasets 26431 r00 and 26432r00. Here dotted vertical lines refer to phase intervals of $135^{\circ}$.

Figs. 1 and 2 these datasets were also obtained in a coronal hole close to and possibly associated with nearby active regions. As before, we expect the measured phases to line up in linear rows, if a fixed time delay is present. Because we expect a linear pattern to be present, we believe it is valid to attempt to fit a line passing through or close to (within the measurement uncertainty) the zero point of phase as in Fig. 3.

The thicker black line in the phase measurement plots shows the result of this linear fit to the measured phase points. From these fitted lines, we can estimate the slopes and, hence, the time delays between the oscillations in the different line pairs. For the Ov 629-Mg X 624 line pair we estimate a time delay of $151 \pm 54 \mathrm{~s}(\approx 6.62 \mathrm{mHz})$, for the O v 629-Si XII 520 line pair a time delay of $211 \pm 64 \mathrm{~s}(\approx 4.74 \mathrm{mHz})$ and for the $\mathrm{Mg} \mathrm{X} \mathrm{624-}$ Si XII 520 line pair a time delay of $121 \pm 33 \mathrm{~s}(\approx 8.26 \mathrm{mHz})$. Again, as before, we can use the fitted lines to shift all of the measured phases up to the horizontal and so produce histograms of the phase distribution. The results of this are shown in Fig. 6. These histograms were created with a bin size that depends on the error in the y-intercept point of the fitted lines for each of the line pairs in Fig. 5. For the O v 629-Mg X 624 line pair, this bin size is $15^{\circ}$, for the O V 629-Si XII 520 line pair it is $60^{\circ}$ and for the Mg X 624-Si XII 520 line pair it is $25^{\circ}$. Looking at the histograms in Fig. 6, and the O V 629-Mg X 624 line pair first, we can see that here the phases are clearly not randomly distributed but produce peaks at intervals of $135^{\circ}$. The peak at $0^{\circ}$ and the peak at $\approx 270^{\circ}$ are at or above the $95 \%$ confidence level shown by the dot-dash line. The peak at $\approx 270^{\circ}$ is, in fact, at $240^{\circ}$, but we believe it to be part of a broader peak of phases distributed between $225^{\circ}$ and $285^{\circ}$, caused by the spread of values around the $270^{\circ}$ point due to measurement uncertainties of $>50-60^{\circ}$ (see errorbars in Fig. 5). The peak at $135^{\circ}$ does not quite reach to the $90 \%$ confidence level. However, if we consider that the peak above the $90 \%$ confidence level at $105^{\circ}$ belongs to a spread of phases around the $135^{\circ}$ point, due to the measurement uncertainties, then we can also say that the $\approx 135^{\circ}$ peak is significant at the $90 \%$ significance level. In the case of the O V 629-Si XII 520 line pair, in Fig. 6 (middle panel), it is clear that there is no obvious distribution of peaks at fixed intervals and the phase distribution is no different than that expected from noise. For the Mg X 624-Si XII 520 line pair in the same figure the separation of the peaks would appear to also be at fixed intervals of $135^{\circ}$, as for the $\mathrm{OV}$ 629-Mg X 624 line pair, although here there is a greater uncertainty. For example, there is no significant peak at

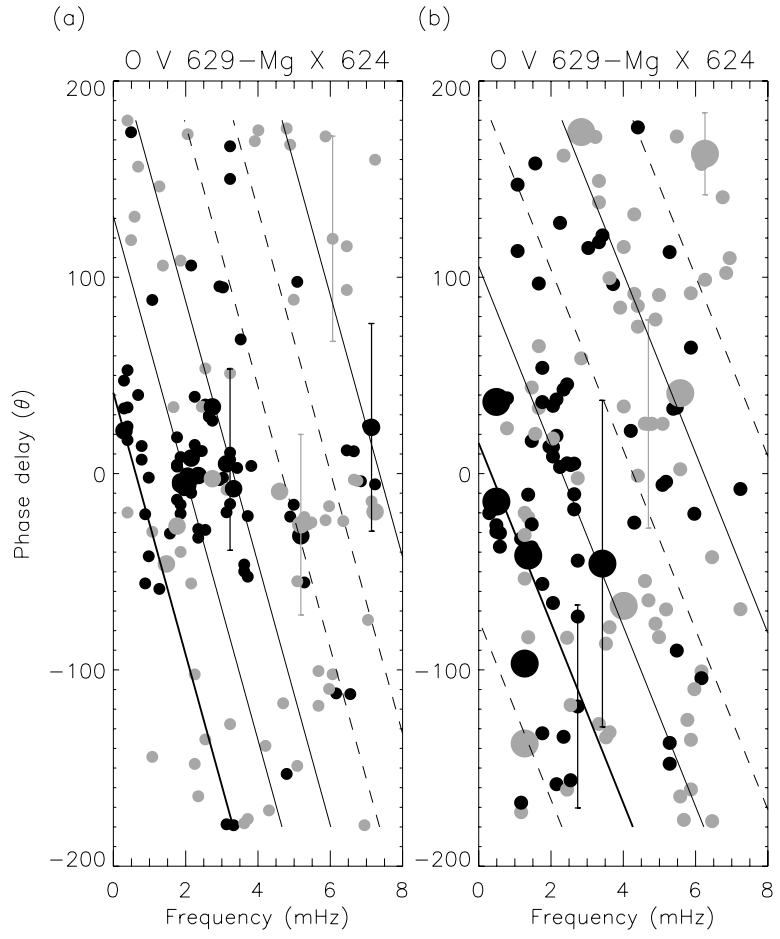

Fig. 7. The same as Fig. 3 presented here for: a) the O v $629-\mathrm{Mg}$ X 624 line pair of the $26435 \mathrm{r} 00$ dataset, and b) the O v $629-\mathrm{Mg}$ X 624 line pair of the combined 26502r00/26503r00 datasets. The solid and dashed lines are spaced at $f / 4$ frequency intervals or $90^{\circ}$ of phase. As before, the dashed lines refer to intervals where there is statistical uncertainty as to the presence of a line at the expected $90^{\circ}$ spacing.

$0^{\circ}$, however, there is a peak above $95 \%$ at $25^{\circ}$ which can probably be attributed to the expected spread of phases around the zero point due to measurement uncertainties. The value at $135^{\circ}$ is not much above the level expected for noise, while we can, perhaps, attribute the peak at $225^{\circ}$ to the spread of values around the expected value of $270^{\circ}$. There is a subsequent significant peak at $405^{\circ}$ at the $90 \%$ confidence level. In Fig. 5, for each of the line pairs, we plot solid lines at the equivalent locations where significant peaks at $135^{\circ}$ intervals were found in the histograms of Fig. 6. At other locations where there were non-significant peaks in the histograms we plot dashed lines. 

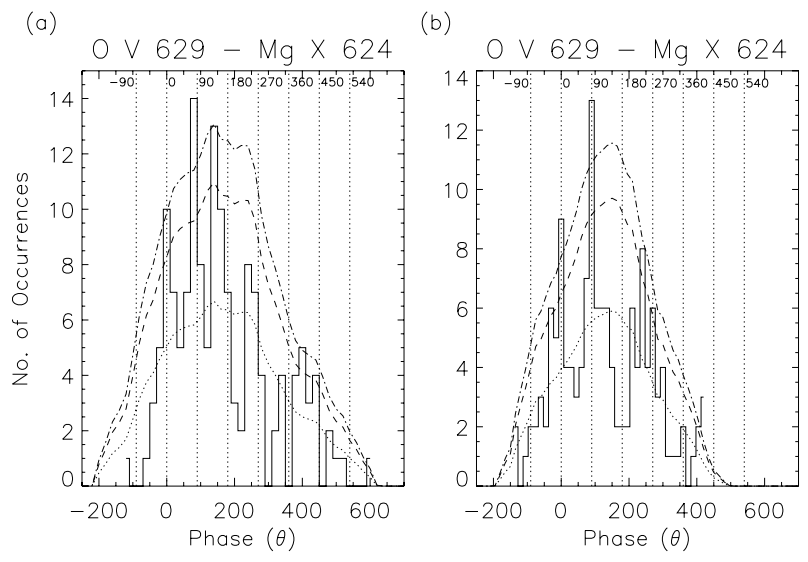

Fig. 8. The same as Fig. 4 presented here for: a) the O V $629-\mathrm{Mg} \mathrm{X}$ 624 line pair of the $26435 \mathrm{r} 00$ dataset, and b) the O v $629-\mathrm{Mg} \mathrm{X} 624$ line pair of the combined 26502r00/26503r00 datasets. Here the dotted vertical lines refer to phase intervals of $90^{\circ}$.

In Figs. 1 and 2 (bottom panels), we show the location of the 26435r00 and combined 26502r00/26503r00 datasets in the Northern and Southern polar coronal hole, respectively. In Fig. 7 we plot the results for the O V 629-Mg X 624 line pairs in these datasets. We only present results of the O V 629-Mg X 624 line pairs here as the Si XII line is too weak and consequently cannot be used for analyses in these polar coronal hole datasets. Following the same procedure of line fitting as for the equatorial coronal hole datasets in Figs. 3 and 5, we calculate time delays of $185 \pm 33 \mathrm{~s}(\approx 5.41 \mathrm{mHz})$ for the 26435 dataset and $127 \pm 26 \mathrm{~s}$ $(7.87 \mathrm{mHz}$ ) for the combined $26502 \mathrm{r} 00 / 26503 \mathrm{r} 00$ datasets.

In Fig. 8, we show, as histograms, the phase distributions for these polar coronal hole datasets. The results for the $26435 \mathrm{r} 00$ dataset are plotted with a bin size of 20 , while the results for the combined $26502 \mathrm{r} 00 / 26503 \mathrm{r} 00$ datasets are plotted with a bin size of 15. Again, the bin size corresponds to the error in the $y$-intercept point of the fitted line. For the $26435 \mathrm{r} 00$ dataset, in Fig. 8, it can be seen that there is a clear $90^{\circ}$ separation, at least between the peaks centred at $0^{\circ}$ and $90^{\circ}$. We can also attribute the peak at $140^{\circ}$ to the spread of phase measurements around the expected value of $180^{\circ}$, as it is clear that it forms part of the broader peak between $120^{\circ}$ and $200^{\circ}$. At $270^{\circ}$ the peak present is not statistically significant but is spaced at the correct and expected spacing of $90^{\circ}$. There is a subsequent significant peak, above the $90 \%$ confidence level at $450^{\circ}$. The spacings of these peaks have been overplotted as the sloping lines in Fig. 7a. Where the peak in the histogram was not statistically significant a dashed line is drawn instead at the expected phase interval of $90^{\circ}$.

For the combined results of $26502 \mathrm{r} 00 / 26503 \mathrm{r} 00$, the histogram in Fig. 8b shows the same spacing of $90^{\circ}$. There are significant peaks at $0^{\circ}, 90^{\circ}$ and $270^{\circ}$ at either the $95 \%$ or $90 \%$ confidence level. Strangely, there is, however, no peak at $180^{\circ}$ which may be just due to the limited number of datapoints used to create these plots. Again, as before, we plot in Fig. $7 \mathrm{~b}$ the sloping lines corresponding to the measured phase spacing of $90^{\circ}$ found in Fig. 8. The dashed lines are again the locations in phase where, in Fig. 8, the peaks in the histograms were not statistically significant.

So, we find from the above that in the equatorial coronal hole location the phases are distributed at spacings of either $90^{\circ}$ (for the combined $26412 \mathrm{r} 00 / 26412 \mathrm{r} 01$ datasets) or $135^{\circ}$ (for the combined $26431 \mathrm{r} 00 / 26432 \mathrm{r} 00$ datasets) and at spacings of $90^{\circ}$ in the polar coronal holes $(26435 \mathrm{r} 00,26502 \mathrm{r} 00 / 26503 \mathrm{r} 00)$. What do these intervals of $90^{\circ}$ and $135^{\circ}$ mean?

It is expected (see O'Shea et al. 2006) that the phases should line up according to the following equation;

$\Delta \phi=2 \pi f T$

with $f$ the frequency and where $\Delta f=1 / T$, with $T$ being the time delay. From this equation, the calculated phase delays are expected to show a rapid change over small values of $\Delta f$, and a variation by a full $360^{\circ}$ every complete $\Delta f$ interval. Therefore, in the plots in Figs. 3, 5 and 7, we should expect the parallel sloping lines to be present at frequency spacings of $\Delta f$ or equivalently at phase spacings of $360^{\circ}$. In fact, as we have seen from the histograms of the phase distributions plotted in Figs. 4, 6 and 8, the phases are present at spacings of either $90^{\circ}$ or $135^{\circ}$ which are equivalent to frequency spacings at $f / 4$ and $3 f / 8$.

Following O'Shea et al. (2006), we can see that the results found here can be interpreted as;

$\Delta \phi=2 \pi(f \pm n \Delta f) T$

where $n$ is the order of the frequency shift, i.e., $0,1,2$, etc., and $\Delta f$ is either $f / 4$ or $3 f / 8$ for the results found here. This suggests that an additional factor is producing a "Doppler effect" upon the (assumed) waves causing the oscillations, producing positive and negative frequency shifts. This additional effect results in a shift in the observed frequencies by multiples of $f / 4$ and $3 f / 8$, as we have seen. The presence of phase delays at these fixed integer frequencies (i.e., $n=0,1,2$, etc.) suggests that the observed oscillations, and, therefore, the waves producing them, are perhaps being influenced by some form of resonant cavity through which the waves pass. We note that a similar result was found by O'Shea et al. (2006) in off-limb polar regions.

Following O'Shea et al. (2006), we note that the measured time delays from the different line pairs may be used to estimate propagation speeds for the waves assumed to be causing the oscillations. In order to calculate the propagation speeds one needs information on the height difference in the atmosphere between the different lines in the line pairs. In O'Shea et al. (2006), these height differences were calculated using limb brightening measurements. Using these same height difference values, but with the time delays measured here in the coronal holes, we show in Table 2 the resulting calculated propagation speeds. It may be clearly seen that the speeds measured at the different line pairs are clearly subsonic in both the equatorial and polar regions. As quoted in O'Shea et al. (2006), the sound speed at the temperature of $\mathrm{MgX}$ is $171 \mathrm{~km} \mathrm{~s}^{-1}$ and $241 \mathrm{~km} \mathrm{~s}^{-1}$ at the temperature of Si XII. Therefore, we see from these results that the propagation speeds in the coronal holes are subsonic and that the waves are therefore likely to be slow magnetoacoustic waves. We note that the propagation speeds measured here are much lower than the values of $154 \pm 18 \mathrm{~km} \mathrm{~s}^{-1}$ measured for the O V 629-Mg X 624 line pair, the value of $201 \pm 17 \mathrm{~km} \mathrm{~s}^{-1}$ measured for the Mg X 629-Si XII 520 line pair and the value of $218 \pm 28 \mathrm{~km} \mathrm{~s}^{-1}$ measured for the O V 629-Si XII 520 line pair in the off-limb polar regions discussed in the paper by O'Shea et al. (2006). One reason for this may be to do with projection effects, that is, the open-field regions assumed to contain the waves may be inclined relative to the line-of-sight. However, in the off-limb data, the waves are assumed to be propagating close to $90^{\circ}$ to the lineof-sight, and yet it is there that we see the larger velocities. In comparision, for the equatorial coronal hole datasets we might expect the waves to be propagating at more acute angles, in the observer's line-of-sight. If we assume that the difference in propagation speeds on and off-disk is not related to projection effects 
Table 2. Propagation speeds measured in the equatorial and polar coronal hole datasets. All speeds are in $\mathrm{km} \mathrm{s}^{-1}$.

\begin{tabular}{lccccl}
\hline \hline & 26412r00/ & 26431r00/ & 26435r00 & 26502r00/ & Height \\
& $26412 \mathrm{r} 01$ & $26432 \mathrm{r} 00$ & & $26503 \mathrm{r} 00$ & difference $(\mathrm{km})$ \\
\hline O V 629 - Mg X 624 & $50 \pm 7$ & $59 \pm 21$ & $48 \pm 9$ & $70 \pm 14$ & 8938 \\
OV 629 - Si XII 520 & $111 \pm 57$ & $110 \pm 33$ & - & - & 23238 \\
Mg X 624 -Si XII 520 & $81 \pm 18$ & $118 \pm 32$ & - & - & 14300 \\
\hline
\end{tabular}

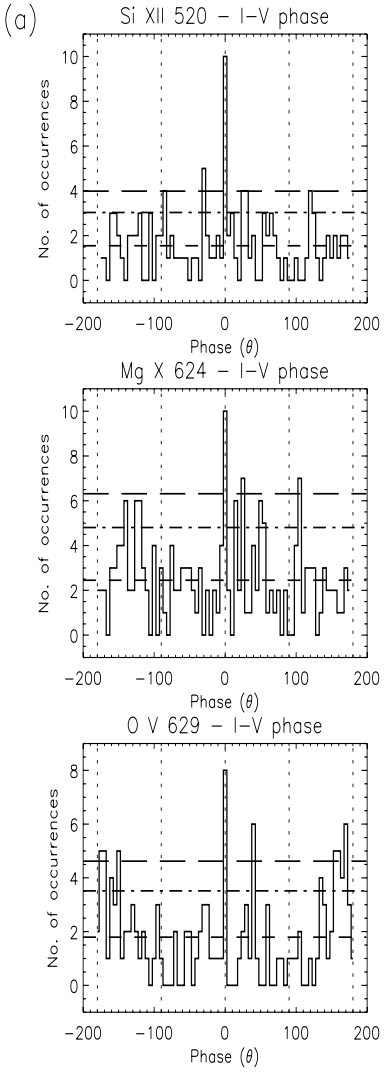

Fig. 9. a) Histograms of flux-velocity (I-V) phase delays, for the different lines as labelled, from the combined equatorial coronal hole datasets. b) Histograms of flux-velocity (I-V) phase delays for the combined polar coronal hole datasets. Si XII is not shown due to its weakness and non-appearance in polar coronal hole locations. The horizontal dashed, dot-dashed and long dashed lines show the $68.3 \%$ (i.e., $1 \sigma$ ), $95 \%(\approx 2 \sigma)$ and $99 \%(\approx 2.6 \sigma)$ confidence levels, respectively, calculated using Monte Carlo simulations with 5000 permutations.

then something else must account for the large difference found. We suggest that the differences in the propagation speeds are related to a more complicated magnetic geometry present in the open field regions on the disk, i.e., in the coronal holes. For example, the equatorial coronal holes are typically associated with decayed active regions (cf. Figs. 1 and 2) and so it is to be expected that the open magnetic field in these regions will not be as uncomplicated as in the polar regions. Hence, the use of the height differences measured using limb brightening and the consequent assumption of a plane parallel atmosphere in the on-disk coronal holes may not be correct.

We have seen from the above results that there is some evidence for there to be a resonant cavity effect taking place in the corona. If such a cavity existed then we might expect to find evidence of standing waves. In order to look for these waves we measure the phase differences between "intensity", that is, radiant flux, and velocity oscillations within a single line, i.e., I-V. If standing waves are present we will expect to find phase values of $90^{\circ}$ in these $\mathrm{I}-\mathrm{V}$ measurements. The combined results for all the equatorial datasets (26412r00, 26412r01, 26431r00, 26432r00) are shown in Fig. 9a, and for all the polar datasets $(26435 \mathrm{r} 00$, 26502r00, 26503r00) in Fig. 9b. The combined results for the polar datasets in Fig. 9b do not show values for Si XII due to the extreme weakness of this line in these areas. On these histograms are plotted dashed, dot-dashed and long dashed lines indicating the confidence levels at $68.3 \%, 95 \%$ and $99 \%$, respectively, calculated using a Monte Carlo (randomisation) simulation with 5000 permutations. The randomisation test used here is based on the null assumption that if there is no structure present in the distribution of the phases, e.g., no tendency to show peaks at $0^{\circ}$, etc., that is, if it is entirely random, then the individual phases values in Fig. 9 could equally well be present at any other phase value between $-180^{\circ}$ and $180^{\circ}$.

If we look first at the results for the equatorial region in Fig. 9a, we can see that there is some evidence for an $\approx 90^{\circ}$ phase peak in the $\mathrm{Mg}$ X 624 line (middle panel), with a peak above the $99 \%$ confidence level at $105^{\circ}$. In addition, there is some evidence for a peak at $-90^{\circ}$, that is, there is a peak at $-120^{\circ}$, above the $90 \%$ significance level, which is likely relevant to the expected $90^{\circ}$ phase peak considering that the average error in these phase measurements is $\approx 60^{\circ}$. (In fact, we find that the error in the phase measurements is $\approx 60^{\circ}$ for all of the lines measured in both the equatorial and polar regions.) In the histogram of the Si XII line, in the top panel of Fig. 9a, we can see that there are also peaks here at $-85^{\circ}$ and at $120^{\circ}$, both just touching the $95 \%$ confidence level. Again, these peaks at $-85^{\circ}$ and $120^{\circ}$ are potentially related to the expected values of $-90^{\circ}$ and $90^{\circ}$ due to the large error and consequential large spread of phase points around the expected $-90^{\circ}$ and $90^{\circ}$ phase positions. If we now look instead at the $\mathrm{O} \mathrm{V}$ line (bottom panel), we can see that it possesses no significant peak ( $>90 \%$ confidence) at or near $-90^{\circ}$ or $90^{\circ}$, unlike the two coronal lines. It does, however, have two broad peaks at $-180^{\circ}$ and $180^{\circ}$ that are clearly absent in the two coronal lines. All three lines, O v, Mg X, Si XII, in contrast, contain strong peaks at zero phase. If we assume that the lines we observe are magnetoacoustic waves propagating upwards then these results suggest a change occurring between the transition region temperature of $\mathrm{OV}$ and the coronal temperatures of the Mg X and Si XII lines. We will return to this point later in Sect. 4.

Looking at Fig. 9b, for the coronal hole datasets, we can see again that for $\mathrm{OV}$ there is a strongly significant peak at $180^{\circ}$ and another significant peak (above 95\%) at $155^{\circ}$. This peak at $155^{\circ}$ can be considered as part of an expected peak at $180^{\circ}$ considering the large measurement uncertainties. For the $\mathrm{Mg}$ X line we again find evidence for standing waves with significant peaks present at $-90^{\circ}\left(>95 \%\right.$ confidence) and at $90^{\circ}(>99 \%$ confidence). Neighbouring significant peaks at $-60^{\circ}$ and $-110^{\circ}$, close to $-90^{\circ}$, are again perhaps explained by a spreading due to measurement uncertainties of the expected $90^{\circ}$ phase value.

We note that, for $\mathrm{MgX}$ in Fig. 9b, there is a peak present above the $99 \%$ confidence level at $150^{\circ}$. Looking again at the plot for the Mg X line in Fig. 9a, we see that there is also a peak present there above the $95 \%$ confidence level at $-140^{\circ}$. These 

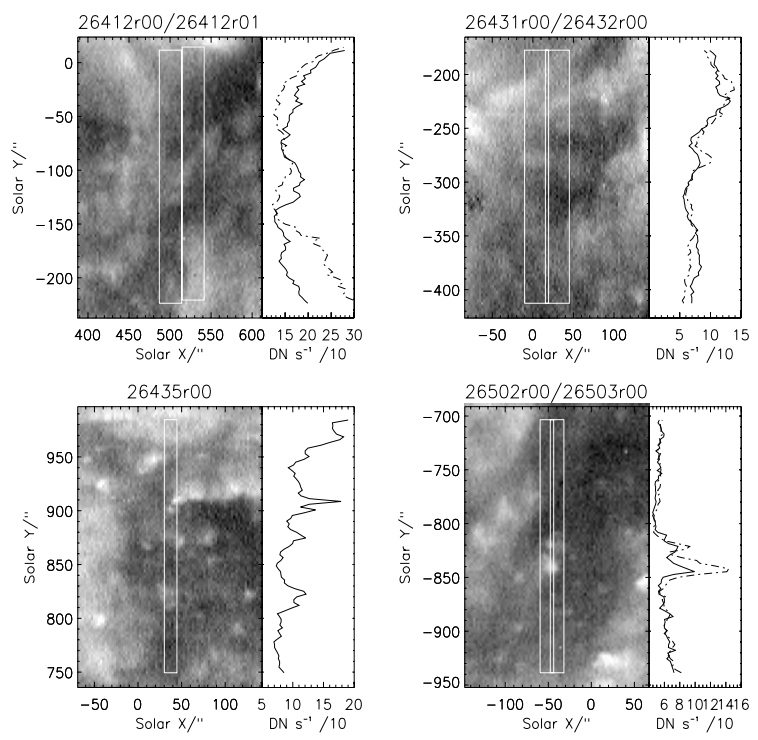

Fig. 10. (Top left) EIT 171 image of 7/12/02 with white boxes showing the area traversed by the 26412r00 (left) and 26412r01 (right) datasets. The summed EIT intensity (in Data Numbers (DN) $\mathrm{s}^{-1}$ ) in these areas for 26412r00 (solid line) and 26412r01 (dot-dashed line). (Top right) EIT 171 images of 10/12/02 showing the area traversed by 26431r00 (right) and the 26432r00 (left). The summed EIT intensity within this area for 26431r00 (solid line) and 26432r00 (dot-dashed line). (Bottom left) EIT 171 images of 11/12/02 showing the area traversed by $26435 \mathrm{r} 00$. The summed EIT intensity within this area for 26435r00 (solid line) (Bottom right) EIT 171 images of 20/12/02 showing the area traversed by $26502 \mathrm{r} 00$ (right) and 26503r00 (left). On the right is shown the summed EIT intensity within this area for $26502 \mathrm{r} 00$ (solid line) and 26503r00 (dot-dashed line).
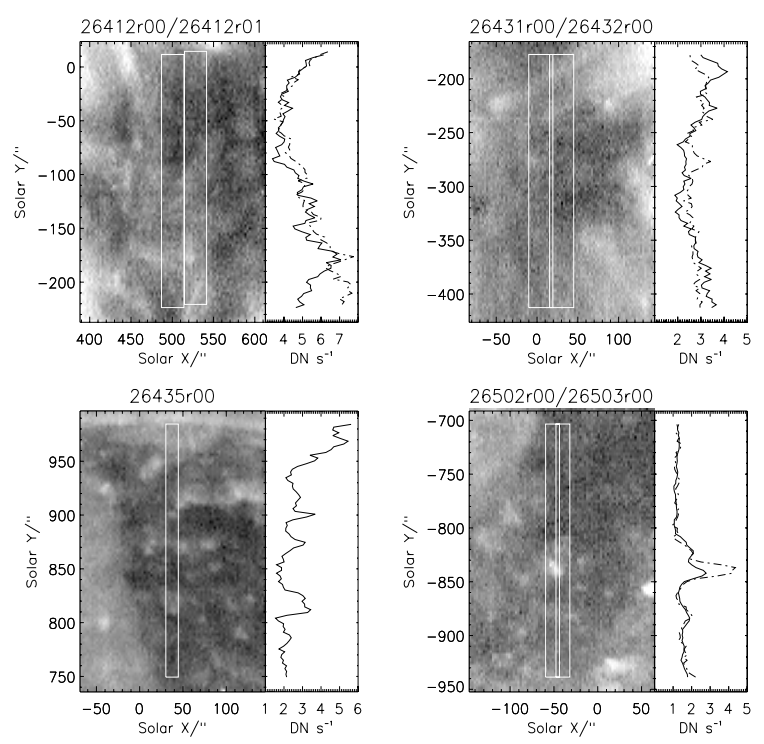

Fig. 11. The equivalent plots as in Fig. 10, showing the results with EIT 284.

results suggest that some waves producing the $-180^{\circ}$ and $180^{\circ}$ phases in O V may also be present at the temperature of $\mathrm{Mg} \mathrm{X}$.

In the off-limb data (O'Shea et al. 2006) the waves producing the $180^{\circ}$ peaks in the I-V histograms of the $\mathrm{O} v$ line were considered to be longitudinal waves, most likely slow magnetoacoustic in nature. This is because it was imagined that if the waves producing $180^{\circ}$ phase values in I-V are longitudinal waves then one might expect the location of maximum intensity to also be the location of the minimum velocity in these types of waves, i.e., at the "crests". Conversely, the peaks at $0^{\circ}$ in the I-V phase plots were considered to be more transverse-like fast magnetoacoustic waves. It was found that the coronal lines of $\mathrm{Mg} \mathrm{X}$ and Si XII showed little sign of $180^{\circ}$ values, but many values clustered around $0^{\circ}$, while the $\mathrm{OV}$ line showed a majority of values clustered at both $-180^{\circ}$ and $180^{\circ}$, with few values at $0^{\circ}$.

In this work, with observations taken on the disk in coronal holes, we observe a similar I-V phase distribution, i.e., $180^{\circ}$ peaks in the $\mathrm{OV}$ line, but none in the coronal lines of $\mathrm{MgX}$ and Si XII, where, in contrast, peaks at $0^{\circ}$ are predominant. This would, perhaps, again suggest that the cooler transition region line of $\mathrm{O} v$ line shows more evidence of longitudinal-type waves. Here, we are observing coronal holes on-disk so most waves should be propagating in the line-of-sight (LOS) of the observer, with some variation due to projection effects., whereas in the offlimb data of O'Shea et al. (2006) most of the waves would have been at or close to $90^{\circ}$ to the LOS. This should not make any difference to the detection of longitudinal-type waves, as if we are able to measure the LOS velocity, then we will always expect the intensity to be $180^{\circ}$ out of phase with the velocity due to the nature of the wave. Interestingly, the $\mathrm{O} v$ line shows a peak at $0^{\circ}$ here that is not present in the I-V plots off-limb. We will discuss these findings more fully in Sect. 4.

So far, therefore, we have seen evidence for propagating and standing slow magnetoacoustic waves in on-disk coronal holes. We know that the waves we infer from oscillations come from coronal hole locations, but we have no information on where exactly in the coronal holes the waves may be originating from. In Figs. 10 and 11, we show close-ups of the EIT images shown in Figs. 1 and 2. It is clear from looking at Figs. 10 and 11 that there is considerable structure present in the coronal holes at the temperatures covered by EIT 171 and 284. In the equatorial coronal holes, the top two images in each of these figures, it can be seen that the region covered by these CDS observations (the white boxes) contains many bright and dark regions. We suggest that the bright regions are the locations of magnetic loops lying across the field-of-view. In the polar coronal hole datasets, the two images in the bottom panels, the structure within the areas covered by the observations also takes the form of many bright and dark areas. However, here there are also a number of welldefined bright points, which are not present in the images for the equatorial datasets. Accompanying these images in Figs. 10 and 11, we plot the variation of the summed EIT intensity within the regions covered by the CDS observations. It can easily be seen from these summed intensity plots that the peaks in the intensity correspond, as expected, to locations of bright patches or bright points within the areas observed.

So, one may ask if the structure found in the coronal hole locations is reflected in the number of significant phases measured along the slit in these locations. That is, are the measured phases distributed evenly along the slit or are they present preferentially in selected locations, perhaps corresponding to the different bright structures found within the coronal holes?

To investigate this we measure the number of phase measurements (in both flux and velocity) obtained at different locations along the observing slit and plot the resulting distribution in the form of a histogram. In Fig. 12, we show the result of this for the equatorial datasets $26412 \mathrm{r} 00$ and 26412r01. Immediately, it is apparent that the phases are not distributed randomly but are clumped at discrete locations along the slit. The bin size for these histograms is $\approx 10^{\prime \prime}$, i.e., equivalent to three of our summed pixels along the observing slit. In Fig. 12a, the results for 26412 r00 are shown together with the variation of the summed intensity 
(a)

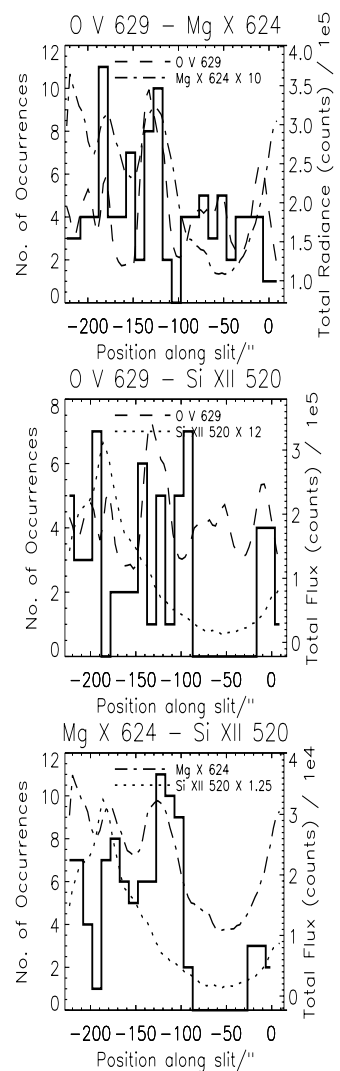

(b)
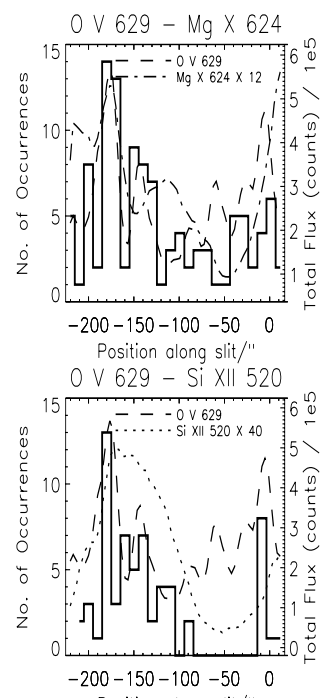

Position along slit/"

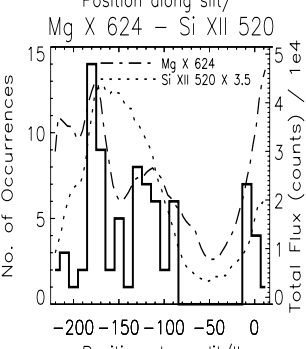

Position along slit/

Fig. 12. a) Histograms showing the number of measured phase delays at each location along the slit for the different line pairs, $\mathrm{O} \mathrm{V}-\mathrm{Mg} \mathrm{X}$, etc., in the 26412r00 dataset. Also plotted are the measurements of total summed intensity along the slit for the relevant line in each case, e.g., $\mathrm{OV}$ and $\mathrm{Mg} \mathrm{X}$ in the upper panels, etc. b) The equivalent results for the 26412 r01 dataset.

along the slit for the $\mathrm{OV}, \mathrm{Mg} \mathrm{X}$ and Si XII lines, depending on the line pairs involved. These summed intensities are the summed intensities over all the 150 time frames in the observations. In the plot for the $\mathrm{OV} 629-\mathrm{Mg}$ X 624 line pair (upper panel) it is apparent that the peaks in the histogram closely coincide with the peaks in the O V summed intensity (the dashed line). Any correlation with the $\mathrm{Mg}$ X line is not so readily apparent. Similarly, in the O V 629-Si XII 520 line pair there is also some correlation of the peaks in the histogram with the peaks in the $\mathrm{OV}$ summed intensity. For the $\mathrm{Mg}$ X 624-Si XII 520 line pair, however, the peaks in the histogram are instead correlated with the summed intensity of the $\mathrm{Mg} \mathrm{X}$. So in each case the histogram showing the occurrences of the phases is correlated with the lower temperature line of the line pair, in this case either OV or MgX. This suggests that the waves are propagating up from more defined structures at the temperatures of these lines.

Note that in these histograms involving Si XII we do not plot any results in the locations where the Si XII intensity falls below a value of 2700 , equivalent to locations where the Signalto-Noise $(\mathrm{S} / \mathrm{N})$ drops to $\approx 3$ or below, e.g., between $\approx-80^{\prime \prime}$ and $\approx-20^{\prime \prime}$ in the plots of O v 629-Si XII 520. In Fig. 12b, similar results in all the line pairs can be seen for the other dataset, 26412 r01, observed in the same equatorial region.

To quantify the degree of correlation found in these datasets we use the Pearson correlation coefficient, which will have a value of $(-1) 1$ for perfect (anti-)correlation and a value of 0 for no correlation. We use the standard IDL routine, CORRELATE

(a)

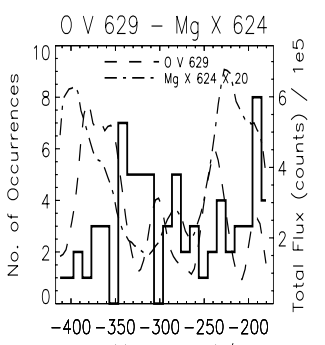

(b)

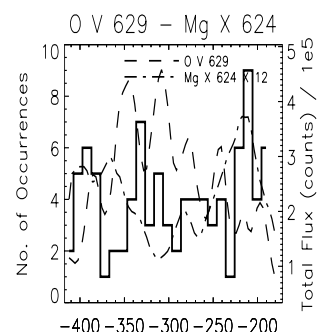

Position along slit/"
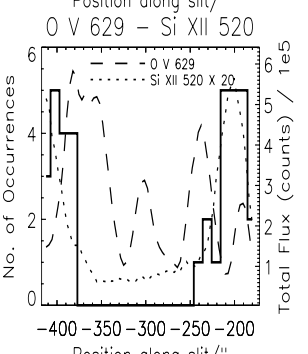

$-400-350-300-250-200$

Position along sit/ $/$

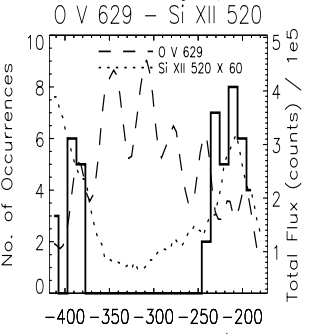

$-400-350-300-250-200$

Position along slit/"

Position along slit/"
Mo X $624-$ Si X 111520
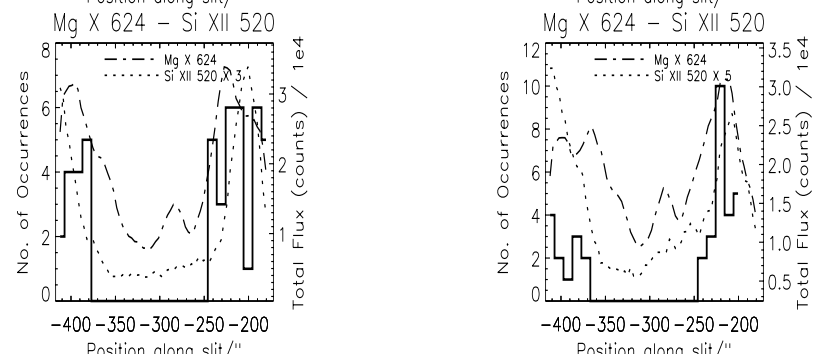

Position along slit/"

Fig. 13. The same as Fig. 12 presented here for: a) dataset 26431r00, and b) dataset $26432 \mathrm{r} 00$

Table 3. Correlation coefficients between the occurence-of-phase histograms and the variation of the summed $\mathrm{OV}$ flux.

\begin{tabular}{cccc}
\hline \hline & Ov 629-/ & Ov 629-/ & Mg X 624-/ \\
& Mg X 624 & Si XII 520 & Si XII 520 \\
\hline 26412r00 & 0.48 & -0.14 & 0.49 \\
$26412 \mathrm{r} 01$ & 0.63 & 0.51 & 0.44 \\
$26431 \mathrm{r} 00$ & -0.31 & - & - \\
$26432 \mathrm{r} 00$ & -0.19 & - & - \\
$26435 \mathrm{r} 00$ & 0.31 & - & - \\
$26502 \mathrm{r} 00$ & 0.49 & - & - \\
$26503 \mathrm{r} 00$ & 0.40 & - & - \\
\hline
\end{tabular}

to calculate this term. The results of this for all datasets measured are shown in Table 3.

It can be see from this table that, except for the O V-Si XII line pair in 26412r00, all line pairs in the 26412r00 and 26412r01 datasets have correlation coefficients close to 0.5 , indicating a significant (if fairly low) level of correlation. The reason for the spurious result of the O V 629-Si XII 520 line pair with a value of -0.13 is likely due to the fact that, while the three peaks in the histogram between $-150^{\prime \prime}$ and $-100^{\prime \prime}$ roughly coincide with the peak in $\mathrm{OV}$ intensity over the same range, there is a drop to a value of only one at two locations, that is, there are two minima, between $-150^{\prime \prime}$ and $-100^{\prime \prime}$. These two minima and three maxima (the peaks) between $-150^{\prime \prime}$ and $-100^{\prime \prime}$ are being compared to a single maximum in $\mathrm{OV}$ in the correlation and it is this that is responsible for the poor quantitative correlation found here. We note that for the O V 629-Si XII 520 and Mg X 624-Si XII 520 line pairs in $26412 \mathrm{r} 00$ and $26412 \mathrm{r} 01$ the correlation was only measured between $\approx-220^{\prime \prime}$ and $\approx-85^{\prime \prime}$ due to the weakness of the Si XII line at other locations. 

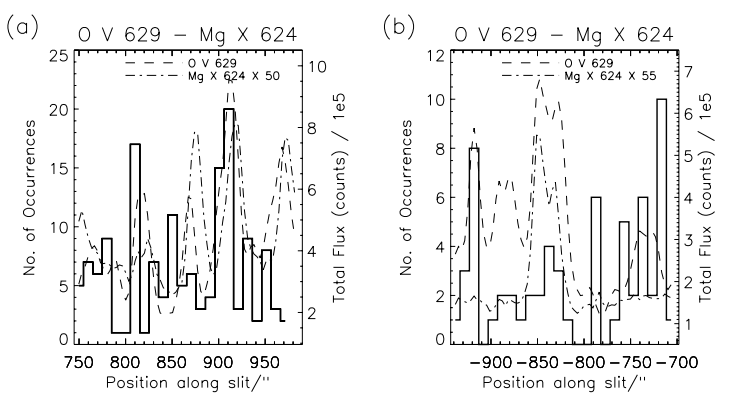

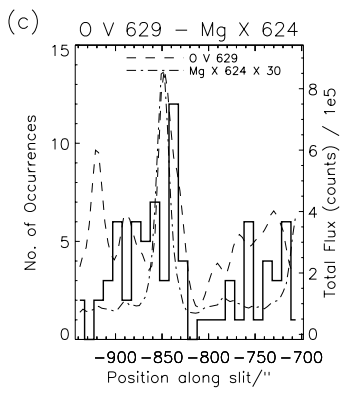

Fig. 14. a) Histograms showing the number of measured phase delays at each location along the slit for the $\mathrm{O} \mathrm{V}-\mathrm{Mg}$ X line pair in the $26435 \mathrm{r} 00$ dataset. Also plotted are the measurements of total summed intensity along the slit for the two relevant lines. b) The equivalent results for the $26502 \mathrm{r} 00$ dataset. c) The equivalent results for the $26503 \mathrm{r} 00$ dataset.
For the other equatorial coronal hole datasets, $26431 \mathrm{r} 00$ and 26432r00, shown in Fig. 13a and b, it can be seen that for the $\mathrm{O}$ v 629-Mg X 624 line pair in each dataset there is a very poor correlation present between the peaks in the histogram and the location of the maximum intensity in $\mathrm{O} v$. This can be confirmed by looking at the low correlation values in Table 3 . For the $\mathrm{O}$ VMg X line pair in 26431r00 in Fig. 13a, there is an obviously poor correlation until $\approx-330^{\prime \prime}$ along the slit at which point the peaks in the $\mathrm{OV}$ intensity again seem to be correlated with the peaks in the histogram. The lack of correlation, however, up to the $-330^{\prime \prime}$ point is enough to affect the correlation coefficient value in Table 3 , giving an anti-correlated value of -0.31 . For the $\mathrm{O} \mathrm{V}-\mathrm{Mg} \mathrm{X}$ line pair in $26432 \mathrm{r} 00$ in Fig. 13b it would appear, in contrast, that there is little or no correlation present between the $\mathrm{O}$ V intensity and the histogram at any point. In this case, there may be more evidence for the histogram to be correlated with the $\mathrm{Mg} \mathrm{X}$ intensity. We do not produce estimates of the correlation coefficient for the other line pairs in these datasets involving Si XII, e.g., O V 629-Si XII or Mg X 624-Si XII 520, due to its weakness over a large part of the observing slit, e.g., between $\approx-380^{\prime \prime}$ to $-250^{\prime \prime}$ in Figs. 13a and b. However, in the locations where the Si XII line is measurable, it can be seen that there is a good visual correlation between the histogram peaks and the intensity values at locations where both O V and Si XII show coincident intensity maxima. This is seen in both the 26431r00 and 26432r00 datasets.

In Fig. 14, we show the results for the 26435r00, 26502r00 and $26503 \mathrm{r} 00$ datasets in the polar coronal holes. Here the Si XII 520 line is so weak that no measurements are possible so only the results from the O v 629-Mg X 624 line pair are shown for each dataset. If we look at Table 3 again, we can again see that there is some correlation between the $\mathrm{O} v$ intensity and the histogram peaks over the full length of the observing slit (the $x$ axis). The correlation is not high but it shows that there is some correspondence, at least, between the location of the maximum occurrence of the phases (the histogram peaks) and the locations where the intensity shows a maximum value.

If we look back now at Figs. 10 and 11, we can compare the results found here with the images of the coronal holes taken with EIT. Looking at the EIT 171 images in the top left panel of Fig. 10, and the area covered by the $26412 \mathrm{r} 00$ and $26412 \mathrm{r} 01$ equatorial datasets, and comparing it to the distribution of the phases along the slit shown in Fig. 12, we can see that the locations in Fig. 12 where the Mg X 624 summed intensity shows a maximum are also approximately the locations where the EIT intensity shows a maximum. We note that EIT 171 and $\mathrm{Mg} \mathrm{X}$ 624 are emitting at approximately the same temperature, close to $1.3 \times 10^{6} \mathrm{~K}$. For example, for the $26412 \mathrm{r} 00$ dataset both the EIT 171 summed intensity (obtained in the area covered by this dataset in time, the white box) in Fig. 10 and the $\mathrm{Mg}$ X summed intensity in Fig. 12a show a peak between $-140^{\prime \prime}$ and $-90^{\prime \prime}$ and another smaller one between $-200^{\prime \prime}$ and $-150^{\prime \prime}$. Looking at Fig. 12, it can be seen that the peaks in the summed $\mathrm{MgX}$ intensity in the O v 629-MgX 624 and Mg X 624-Si XII 520 line pair plots are also the locations of peaks in the phase histograms. This, therefore, strongly suggests that the phase delays measured in this work, and the waves that are producing them, are originating preferentially in the bright locations along the slits. Unfortunately, we do not possess images, raster or otherwise, of $\mathrm{OV}$ in the same location, so it is not possible to do a similar comparison with the variation of the summed $\mathrm{O} V$ intensity in Fig. 12a. Similar results can be seen for the 26412r01 dataset in Fig. 12b, if we compare them to the EIT 171 and EIT 284 images in Figs. 10 and 11. Here the peaks of the phase histograms in the Mg X 624-Si XII 520 line pair can again be seen to correspond to the locations of brightenings along the slit in the EIT 171 image in Fig. 10 (top left), e.g., between $-140^{\prime \prime}$ and $-90^{\prime \prime}$. In Fig. 11, the EIT 284 summed intensity in the area covered by the $26412 \mathrm{r} 01$ dataset shows a peak at $\approx-180^{\prime \prime}$, associated with a large bright spot in the image, which corresponds with a peak found in the phase delay histograms at the same location for the O v 629-Mg X 624, O v 629 -Si XII 520 and Mg X 624Si XII 520 line pairs.

As mentioned previously, the phase histograms of the O v 629-Mg X 624 line pair in Fig. 13 for the 26431r00 and 26432r00 equatorial datasets show a very limited correlation with the maxima found in the $\mathrm{OV}$ and $\mathrm{MgX}$ summed intensities. However, we found that the O V $629-\mathrm{Mg}$ X 624 line pairs in 26431r00 and 26432r00 showed some correspondence with the maxima in either the $\mathrm{OV}$ or $\mathrm{Mg} \mathrm{X}$ line between $-300^{\prime \prime}$ and $-180^{\prime \prime}$. We note that this region corresponds with a general increase in brightening in the EIT 171 image shown in Fig. 10 (top right).

Looking now at the polar coronal hole results, the O V 629$\mathrm{Mg}$ X line pairs shown in Fig. 14, it can be seen that the two main peaks of the $26435 \mathrm{r} 00$ phase histogram in Fig. 14a between $800^{\prime \prime}$ and $820^{\prime \prime}$ and $890^{\prime \prime}$ and $920^{\prime \prime}$ correspond exactly to the locations of large brightenings seen in the EIT 171 and EIT 284 summed intensities in Figs. 10 and 11 (bottom left). These brightenings can be seen, in the EIT 171 and 284 images, to be due to small regions similar to bright points present in these polar coronal locations. In Figs. 10 and 11 the area covered by the $26502 \mathrm{r} 00$ and $26503 \mathrm{r} 00$ datasets contains a large bright area between $\approx-850^{\prime \prime}$ to $810^{\prime \prime}$. This shows up in the summed EIT 171 and 284 intensity as two peaks. If we look at the range of $\approx-850^{\prime \prime}$ to $-810^{\prime \prime}$ in Fig. $14 \mathrm{~b}$ and c we can see that, especially for the $26503 \mathrm{r} 00$ dataset, this location corresponds to a peak in the phase histogram. In the case of $26502 \mathrm{r} 00$ there is a peak in the histogram in this region but other locations along the slit show a larger number of phase occurrences.

In Fig. 15 we show, for the 26412r00 and 26412r01 datasets, the different frequencies at which phase measurements were obtained in radiant flux (circles) and velocity oscillations (squares). This plot is equivalent to the one shown in Fig. 12, except here 
(a)

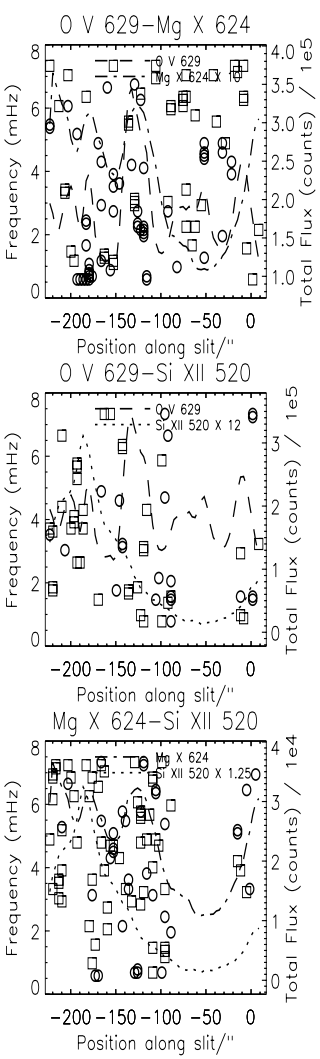

(b)

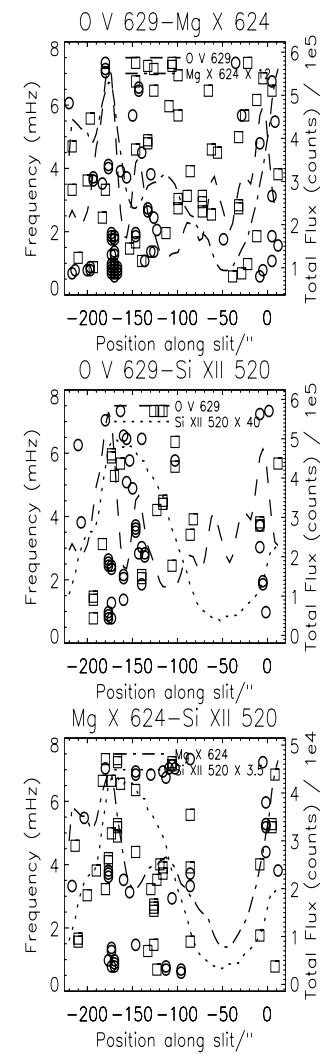

Fig. 15. a) Plots showing the location of the frequencies, corresponding to the measured phase delays, for the different line pairs, O v $629-\mathrm{Mg}$ X 624 , etc., in the $26412 \mathrm{r} 00$ dataset. Measurements obtained from radiant flux oscillations are plotted as circles while those obtained from velocity oscillations are plotted as squares. Also plotted are the measurements of total summed intensity along the slit for the relevant line in each case, e.g., $\mathrm{OV}$ and $\mathrm{Mg} \mathrm{X}$ in the plots in the upper panels, etc. b) The equivalent results for the $26412 \mathrm{r} 01$ dataset.

each individual phase measurement is represented by the corresponding frequency at which it was measured. If we look at the plots in Fig. 15, it is immediately clear that the measured oscillations are distributed over the full measurable frequency range, i.e., from $\approx 0.6 \mathrm{mHz}$ up to $\approx 7.5 \mathrm{mHz}$. Also, if we look at the $\mathrm{O} \mathrm{V}$ 629-Mg X 624 line pair of 26412r00 in Fig. 15a, it can be seen that at certain locations, e.g., at $-120^{\prime \prime}$, there are a number of significant frequencies simultaneously present, covering a limited range. These clusters of frequencies are found in both the intensity and velocity oscillations. These clusters of frequencies at certain locations indicate that the waves producing the phase delays are present at a spectrum of frequencies at specific locations. If we look at the locations at where these clusters occur and compare them to the peaks in the summed $\mathrm{OV}$ and $\mathrm{Mg} \mathrm{X}$ intensities, and to the peaks in the phase histograms in Fig. 12, it is clear that there is a tendency for this frequency clustering to occur at the locations of brightness in the observing field-ofview. We note that the same pattern of clustering is found in the other equatorial and polar coronal hole datasets, which we do not include here due to lack of space.

So, from our study of Figs. 10-15, we have found evidence for the measured phase delays and their associated oscillation frequencies to be preferentially located in bright regions in the observed field-of-view. If we look at the top panels of Figs. 10 and 11 , for the equatorial coronal hole locations, it can be seen that there are many bright locations within the observed fieldof-view. We suggest that these bright areas are the locations of magnetic structures, possibly loops, or collections of loops, associated with the adjacent active regions in these equatorial coronal hole regions (cf. Figs. 1 and 2). In the polar coronal hole regions, the bottom panels of Fig. 10 and Fig. 11, the bright regions associated with the phase delays are more limited in size and are likely due to clusters of flux tubes, perhaps at network boundaries. As we have seen, there is a marked preference for the waves we infer from this work to propagate in these brighter regions in the observed field-of-view.

\section{Discussion and conclusions}

In this work we have found evidence for propagating slow magnetoacoustic waves in equatorial and polar coronal open field regions. We find the waves to have similar speeds of propagation in both of these on-disk regions. We note that the speeds found by us on-disk here are lower than those found off-limb in the polar coronal holes (O'Shea et al. 2006). In both cases, on-disk and off-limb, the same height differences calculated from limb brightenings have been used to calculate the propagation speeds. The difference in speeds between the open field regions on and off-disk may suggest that the magnetic structure in the open-field regions on disk may not be as uncomplicated as that found offlimb. That is, the use of the height differences calculated from limb brightenings may not be correct in relation to the more complicated magnetic topology present on-disk. Unfortunately, we do not possess an alternative method to calculate average height differences between the formation heights of the different lines on the disk.

The finding that the different line pairs in the equatorial and polar coronal holes possess fixed time delays, i.e., within a fixed range, further suggests that the propagation speeds possess a discrete range of speeds in these regions. The discovery here that the measured phase delays are spaced at integer intervals of frequency, $f / 4\left(90^{\circ}\right)$ and $3 f / 8\left(135^{\circ}\right)$, in addition, strongly suggests the presence of a resonant cavity effect in these open-field ondisk regions. This is a similar result to that found by O'Shea et al. (2006) in off-limb polar regions. In support of the evidence that resonant cavities may be present, we find phases delays of $90^{\circ}$ in I-V phase measurements. These phase delays of $90^{\circ}$ indicate the presence of standing waves. These $90^{\circ}$ measurements are, however, only measured in the coronal lines of $\operatorname{Mg~X~} 624$ and Si XII 520, perhaps indicating that standing waves, and, hence, any resonant cavity effect are restricted to coronal temperatures.

If we consider that the magnetic field is open in the different coronal hole regions examined, we might then expect that at coronal temperatures any flux tube would be too large to support standing waves, e.g., those produced by reflection at the transition region/corona interface. However, we note that in equatorial coronal hole locations closed loops and open loops can be present together. In fact, closed loops may be present in the equatorial coronal hole locations shown in EIT 171 data at the top of Fig. 1. We note that standing waves occur in closed structures such as loops, provided the wave has had time to travel the entire length of the loop and back again. For shorter time lengths the wave will not have reached the ends of the loop, where linetying in the dense lower atmosphere causes reflection, and so the wave propagates freely as in an open flux tube. In such a scenario, we may expect to see evidence of both propagating and standing waves.

From the I-V phase measurements we find that the transition region line of $\mathrm{O} V$ contains numerous phase values of $-180^{\circ}$ and $180^{\circ}$. Following Brynildsen et al. (1999), we could interpret these phases at $180^{\circ}$ as being due to upwardly propagating 
waves at the transition region temperature of $\mathrm{O} v$. However, $\mathrm{O} \mathrm{v}$ also contains a large peak in the $\mathrm{I}-\mathrm{V}$ phases at $0^{\circ}$, something also seen in the two coronal lines of $\mathrm{Mg} \mathrm{X}$ and Si XII. If we accept that the phases at $\pm 180^{\circ}$ are due to upwardly propagating waves, then we must also accept that the phases at $0^{\circ}$ are due to downwardly propagating waves. However, we find no evidence for downwardly propagating waves in this work and the idea that these downwardly propagating waves may be present at the temperatures of O V, Mg X and, especially, Si XII does not seem likely. We note that the standard interpretation of the $180^{\circ}$ phases found in I-V plots is that, if the maximum in the "intensity" is correlated with the minimum in the Doppler velocity, i.e., a "blueshift", then the wave producing the oscillations is propagating upwards. However, many measurements of Doppler velocity are, in reality, measurements of relative velocity and not absolute velocity (cf. Sect. 2). Therefore, not all minima measured in the relative Doppler velocities measured here are due to actual blueshifted plasma and are merely registering a localised reduction in the measured velocity. If the waves we are measuring are sound-like longitudinal waves, then it is to be expected that the velocity will show a minimum at the location of maximum intensity, that is, at the "crests" of these waves where the density of the plasma in the wave will be greatest. Conversely, where the velocity is at a maximum in the locations in between the crests, the intensity will be at a minimum. Therefore, we see from this, that for a longitudinal wave, the presence of a $180^{\circ}$ phase difference between I and V may come from the nature of the wave itself.

The peaks seen at $0^{\circ}$ found in the $\mathrm{O} \mathrm{V}, \mathrm{Mg} \mathrm{X}$ and Si XII lines in Fig 9 can then, conversely, be considered as being due to more transverse-like waves. We note that Cooper et al. (2003) have shown that oscillations in even incompressible transverse-like kink waves may be observed depending on the projection angle of the LOS. We, therefore, note that the transverse-like waves inferred here may be fast magnetoacoustic waves, propagating at shallow angles $\left(\ll 90^{\circ}\right)$ to the LOS, with the resulting Doppler velocity maxima occurring in phase with perturbations of plasma density and, hence, the intensity. However, if these fast magnetoacoustic waves are indeed present, we see no evidence of them from the measurements of propagation speeds in Table 2, which indicate only the presence of propagating slow magnetoacoustic waves. However, we noted previously that the height differences used here are an estimation obtained from limb brightening and, therefore, they may not be entirely appropriate for on-disk observations. It is possible, then, that the values in Table 2 seriously underestimate the true speeds present in the coronal holes. However, it must also be noted that, in order to account for the failure to measure fast magnetoacoustic waves, i.e., supersonic speeds, the measured heights would have to be incorrect by factors of between $2-4$.

In Fig. 9 we base our view that fast waves are present on the peaks found in each line at $0^{\circ}$. However, these peaks only have values of between 8 and 10, suggesting a smallish numbers of occurrences of these fast wave and many more other waves present with phases between $-180^{\circ}$ and $+180^{\circ}$. This suggests a mixture of waves is present and that the propagation speeds measured by us merely represent an averaged value due to a mixing of fast and slow waves. An alternative scenario for the fact we do not find evidence of propagation speeds appropriate to fast mode waves, and yet see evidence for these fast mode waves in Fig. 9, is that the assumed fast magnetoacoustic waves are not propagating. Indeed, this scenario would fit well with our discovery of standing waves at coronal temperatures. However, all of this is merely speculative. In conclusion, from the available evidence (e.g., the propagation speeds in Table 2), we must conclude that the propagating waves we measure are slow magnetoacoustic waves. This would tally with the results found by O'Shea et al. (2006).

By plotting the location of the measured phases as a function of position along the observing slit, it has been possible to establish a link (correlation) between the brightenings seen at different locations along the slit and the location of the bulk of the propagating waves, i.e., the location of the measured phases. We suggest that, in the equatorial coronal holes, the brightenings along the slit are the locations of open magnetic flux tubes close to active regions and, in the polar coronal holes, that they are clusters of unipolar magnetic field lines on network boundaries. In the polar coronal holes the brightenings are less diffuse and take the form of discrete bright points. We suggest here that these bright points may be the coronal funnels mentioned by Tu et al. (2005). These results suggest that the waves we infer from these measurements are propagating along the open magnetic field in the two regions we investigate, as indeed might be expected for magnetoacoustic waves.

Finally, we find evidence that, at bright regions along the slit, the measured phases tend to occur at a spectrum of frequencies. This clustering of measured phases over a limited spectrum of frequencies perhaps suggests the presence of discrete propagating wave packets.

We note that the magnetoacoustic waves inferred from this work can provide significant momentum and energy for the heating of coronal holes and the acceleration of the solar wind. Coronal heating and acceleration of the high- and low-speed solar wind in the open field region by dissipation of fast and slow MHD waves, through the formation of MHD shocks, was studied with 1D MHD models (Orta et al. 2003; Suzuki 2004). Orta et al. (2003) concluded that large amplitude MHD waves that steepen into fast and slow MHD shocks in low-beta regions could be a viable mechanism for coronal heating and wind acceleration in regions of open magnetic field lines. Similarly, Suzuki (2004) found that linearly polarised fast and slow magnetosonic waves traveling upwardly along the magnetic field eventually form fast switch-on shock trains and hydrodynamical shock trains, respectively, to heat and accelerate the plasma. Our observations can be used to constrain the models and test their validity.

From our observations it is clear that the velocity amplitudes are quite small, thus the linear theory may well be applicable. The energy carried by the slow magneto-acoustic waves can be estimated as $\rho\left[(\delta v)^{2} / 2\right] v_{\mathrm{s}}$, in WKB approximation, where $\delta v$ is the wave velocity amplitude, and $v_{\mathrm{s}}$ is the propagation speed. At coronal heights if we assume the average propagation speed of $\approx 100 \mathrm{~km} \mathrm{~s}^{-1}$ (see Table 2), $\delta v=10 \mathrm{~km} \mathrm{~s}^{-1}$ (a typical value), and the density at the base of coronal hole $\rho=5 \times 10^{-15} \mathrm{~g} \mathrm{~cm}^{-3}$, we get a wave energy flux of $\approx 2.5 \times 10^{4} \mathrm{erg} \mathrm{cm}^{-2} \mathrm{~s}^{-1}$. This is lower than the energy flux requirement of a coronal hole with a high speed wind $\left(\approx 8 \times 10^{5} \mathrm{erg} \mathrm{cm}^{-2} \mathrm{~s}^{-1}\right)$, but non-WKB effects may enhance the energy flux somewhat.

These slow waves can damp quickly via compressive viscosity and thermal conduction close to the sun $\left(r<2 R_{\odot}\right)$, or via Landau damping in the collisionless plasma further out. The prevailing view based on Doppler shift observations (Athay \& White 1978) is that the slow wave energy flux in the chromosphere is not sufficient to balance the coronal energy losses. Evidently, the sound waves launched in the photosphere undergo strong reflection. This is consistent with the small energy flux as calculated above. However, if the observed nonthermal Doppler broadening of $20-60 \mathrm{~km} \mathrm{~s}^{-1}$ of heavy ion emission lines 
is attributed to slow waves (Porter et al. 1994), or most of the slow wave spectrum is undetected as suggested by Tsiklauri \& Nakariakov (2001), than it is possible that slow waves may contribute significantly to coronal heating.

We conclude from this work that propagating slow magnetoacoustic waves are present in coronal hole locations at the equator and at the poles. We find that these waves are propagating preferentially in regions of increased brightness in the coronal holes that we consider to be locations of concentrated magnetic field. Measurements of I-V phases indicate the presence of standing waves in these coronal hole locations at coronal temperatures. Evidence for a resonant cavity effect is provided by the tendency for the phase delay measured between the different line pairs to occur at fixed integer frequency intervals of $f / 4$ $\left(90^{\circ}\right.$ of phase) and $3 f / 8$ ( $135^{\circ}$ of phase), which may be linked to the presence of standing waves.

Acknowledgements. We would like to thank the full CDS team for their help in obtaining and reducing the data. CDS and EIT are part of SOHO, the Solar and Heliospheric Observatory, which is a project of international cooperation between ESA and NASA. This work was supported in part by a PRTLI research grant for Grid-enabled Computational Physics of Natural Phenomena (Cosmogrid) and PPARC grants PPA/G/S/2002/00020 and PP/D001129/1. We wish to thank the Royal Society and the British Council for funding visits between Armagh Observatory and the Indian Instutite of Astrophysics.

\section{References}

Athay, R. G., \& White, O. R. 1978, ApJ, 226, 1135.

Banerjee, D., O’Shea, E., Doyle, J. G., \& Goossens, M. 2001, A\&A, 380, L39 Brynildsen, N., Kjeldseth-Moe, O., Maltby, P., \& Wilhelm, K. 1999, ApJ, 517, L159

Cooper, F. C., Nakariakov, V. M., \& Tsiklauri, D. 2003, A\&A, 397, 765

DeForest, C. E., \& Gurman, J. B. 1998, ApJ, 501, L217

Davila, J. M. 1985, ApJ, 291, 328

Harrison, R. A., Sawyer, E. C., Carter, M. K., et al. 1995, Sol. Phys., 162, 233

Krieger, A. S., Timothy, A. F., \& Roelaf, E. C. 1973, Sol. Phys., 29, 505

Munro, R. H., \& Withbroe, G. L. 1972, ApJ, 176, 511

Nakariakov, V. M., Melnikov, V. F., \& Reznikova, V. E. 2003, A\&A, 412, L7

Ofman, L., Romoli, M., Poletto, G., Noci, G., \& Kohl, J. L. 1997, ApJ, 491, L111

Ofman, L., Romoli, M., Poletto, G., Noci, G., \& Kohl, J. L. 2000, ApJ, 529, 592

Orta, J. A., Huerta, M. A., \& Boynton, G. C. 2003, ApJ, 596, 646

O'Shea, E., Banerjee, D., \& Doyle, J. G. 2006, A\&A, 452, 1059

Porter, L. J., Klimchuk, J. A., \& Sturrock, P. A. 1994, ApJ, 435, 482

Suzuki, T. K. 2004, MNRAS, 349, 1227

Teriaca, L., Poletto, G., Romoli, M., \& Biesecker, D. A. 2003, ApJ, 588, 566

Tsiklauri, D., \& Nakariakov, V. M. 2001, A\&A, 379, 1106

Tu, C.-Y., Zhou, C., Marsch, E., et al. 2005, Sci, 308, 519

Wilhelm, K., Dammasch, I. E., Marsch, E., \& Hassler, D. M. 2000, A\&A, 353, 749

Xia, L. D., Marsch, E., \& Wilhelm, K. 2004, A\&A, 424, 1025 